\title{
MULTIPLE ORGAN FAILURE DIAGNOSIS USING ADVERSE EVENTS AND NEURAL NETWORKS
}

\author{
Álvaro Silva \\ Hospital Geral de Santo António \\ Porto, Portugal \\ a.moreirasilva@mail.telepac.pt
}

\author{
Paulo Cortez \\ DSI, Universidade do Minho \\ Guimarães, Portugal \\ pcortez@dsi.uminho.pt
}

\author{
Manuel Santos \\ DSI, Universidade do Minho \\ Guimarães, Portugal \\ mfs@dsi.uminho.pt
}

\author{
Lopes Gomes \\ Inst. de Ciências Biomédicas Abel Salazar \\ Porto, Portugal \\ cardiologia.hgsa@mail.telepac.pt
}

\author{
José Neves \\ DI, Universidade do Minho \\ Braga, Portugal \\ jneves@di.uminho.pt
}

Key words: Intensive Care Medicine, Classification, Clinical Data Mining, Multilayer Perceptrons.

Abstract: $\quad$ In the past years, the Clinical Data Mining arena has suffered a remarkable development, where intelligent data analysis tools, such as Neural Networks, have been successfully applied in the design of medical systems. In this work, Neural Networks are applied to the prediction of organ dysfunction in Intensive Care Units. The novelty of this approach comes from the use of adverse events, which are triggered from four bedside alarms, being achieved an overall predictive accuracy of $70 \%$.

\section{Introduction}

Scoring the severity of illness has become a daily routine practice in Intensive Care Units (ICUs), with several metrics available, such as the Acute Physiology and Chronic Health Evaluation System (APACHE II) or the Acute Physiology Score (SAPS II), just to name a few (Teres and Pekow, 2000). Yet, most of these prognostic models (given by Logistic Regression) are static, being computed with data collected within the first 24 hours of a patient's admission. This will produce a limited impact in clinical decision making, since there is a lack of accuracy of the patient's condition, with no intermediate information being used.

On the other hand, the Clinical Data Mining is a rapidly growing field, which aims at discovering patterns in large clinical heterogeneous data (Cios and Moore, 2002). In particular, an increasing attention has been set over the use of Neural Networks (connectionist models that mimic the human central nervous system) in Medicine, with the number of publications growing from two in 1990 to five hundred in 1998 (Dybowski, 2000).

The interest in Data Mining arose due to the rapid emergence of electronic data management methods, holding valuable and complex information (Hand et al., 2001). However, human experts are limited and may overlook important details, while tecniques such as Neural networks have the potential to solve some of these hurdles, due to capabilities such as nonlinear learning, multi-dimensional mapping and noise tolerance (Bishop, 1995).

In ICUs, organ failure diagnosis in real time is a critical task. Its rapid detection (or even prediction) may allow physicians to respond quickly with therapy (or act in a proactive way). Moreover, multiple organ dysfunction will highly increase the probability of the patient's death.

The usual approach to detect organ failure is based in the Sequential Organ Failure Assessment (SOFA), a diary index, ranging from 0 to 4 , where an organ is considered to fail when its SOFA score is equal or higher than 3 (Vincent et al., 1996). However, this index takes some effort to be obtained (in terms of time and costs).

This work is motivated by the success of previous applications of Data Mining techniques in ICUs, such as predicting hospital mortality (Santos et al., 2002). The aim is to study the application of $\mathrm{Neu}$ ral Networks for organ failure prediction (identified by high SOFA values) of six systems: respiratory, coagulation, liver, cardiovascular, central nervous and renal. Several approaches will be tested, using different feature selection, training and modeling configurations. A particular focus will be given to the use of daily intermediate adverse events, which can be automatically obtained from four hourly bedside measurements, with fewer costs when compared to the SOFA score.

The paper is organized as follows: first, the $I C U$ clinical data is presented, being preprocessed and 
transformed into a format that enables the classification task; then, the neural models for organ failure diagnosis are introduced; next, a description of the different experiments performed is given, being the results analyzed and discussed; finally, closing conclusions are drawn.

\section{Materials and Methods}

\subsection{Clinical Data}

In this work, a part of the EURICUS II database (www. frice.n1) was adopted which contains data related to 5355 patients from 42 ICUs and 9 European Union countries, collected during a period of 10 months, from 1998 to 1999 . The database has one entry (or example) per each day (with a total of 30570), being its main features described in Table 1:

- The first six rows denote the SOFA values (one for each organ) of the patient's condition in the previous day. In terms of notation, these will be denoted by $S O F A_{d-1}$, where $d$ represents the current day.

- The case mix appears in the next four rows, an information that remains unchanged during the patient's internment, containing: the admission type (1 - Non scheduled surgery, 2 - Scheduled surgery, 3 - Physician); the admission origin (1 - Surgery block, 2 - Recovery room, 3 - Emergency room, 4 - Nursing room, 5 - Other ICU, 6 - Other hospital, 7 - Other sources); the SAPSII score (a mortality prediction index, where higher values suggest a high death probability) and the patient's age. Figure 1 shows the frequency distributions of these attributes.

- Finally, the last four rows denote the intermediate outcomes, which are triggered from four monitored biometrics: the systolic Blood Pressure (BP); the Heart Rate (HR); the Oxygen saturation (O2); and the URine Output (UR). A panel of EURICUS II experts defined the normal ranges for these four variables (Tables 2 and 3). Each event (or critical event) is defined as a binary variable, which will be set to 0 (false), if the physiologic value lies within the advised range; or 1 (true) else, according to the time criterion.

Before attempting modeling, the data was preprocessed, in order to set the desired classification outputs. First, six new attributes were created, by sliding the $S O F A_{d-1}$ values into each previous example, since the intention is to predict the patient's condition $\left(S O F A_{d}\right)$ with the available data at day $d$ (SOF $A_{d-1}$, case mix and adverse events). Then, the last day of the patient's admission entries were discarded (remaining a total of 25309), since in this cases, no $S O F A_{d}$ information is available. Finally, the new attributes were transformed into binary variables, according to the expression:

$$
\begin{array}{ll}
0, \text { if } S O F A_{d}<3 & \begin{array}{l}
\text { (false, no organ failure) } \\
\text { (true, organ dysfunction) }
\end{array}
\end{array}
$$

\subsection{Neural Networks}

In MultiLayer Perceptrons, one of the most popular Neural Network architectures, neurons are grouped into layers and only forward connections exist (Bishop, 1995). Supervised learning is achieved by an iterative adjustment of the network connection weights (the training procedure), in order to minimize an error function, computed over the training examples (cases).

The state of a neuron $\left(s_{i}\right)$ is given by (Haykin, 1999):

$$
s_{i}=f\left(w_{i, 0}+\sum_{j \in I} w_{i, j} \times s_{j}\right)
$$

where $I$ represents the set of nodes reaching node $i, f$ the activation function (possibly of nonlinear nature), $w_{i, j}$ the weight of the connection between nodes $j$ and $i$ (when $j=0$, it is called bias); and $s_{1}=x_{1}, \ldots, s_{n}=x_{n}$, being $x_{1}, \ldots, x_{n}$ the input vector values for a network with $n$ inputs.

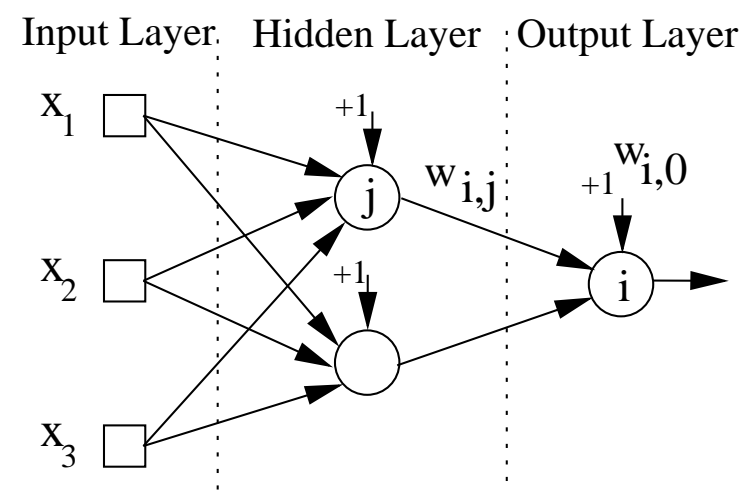

Figure 2: A fully connected network with 3 inputs, 2 hidden nodes, 1 output and bias connections.

All experiments reported in this work will be conducted using a neural network object oriented programming environment, developed in JAVA.

Fully connected Multilayer Perceptrons with bias connections, one hidden layer (with a fixed number of hidden nodes) and logistic activation functions $\left(f(x)=\frac{1}{1+e^{-x}}\right)$ were adopted for the organ failure classification (Figure 2). Only one output node is used, since each organ system will be modeled by a different network. This splitting is expected to facilitate the Neural Network learning process. Therefore, 

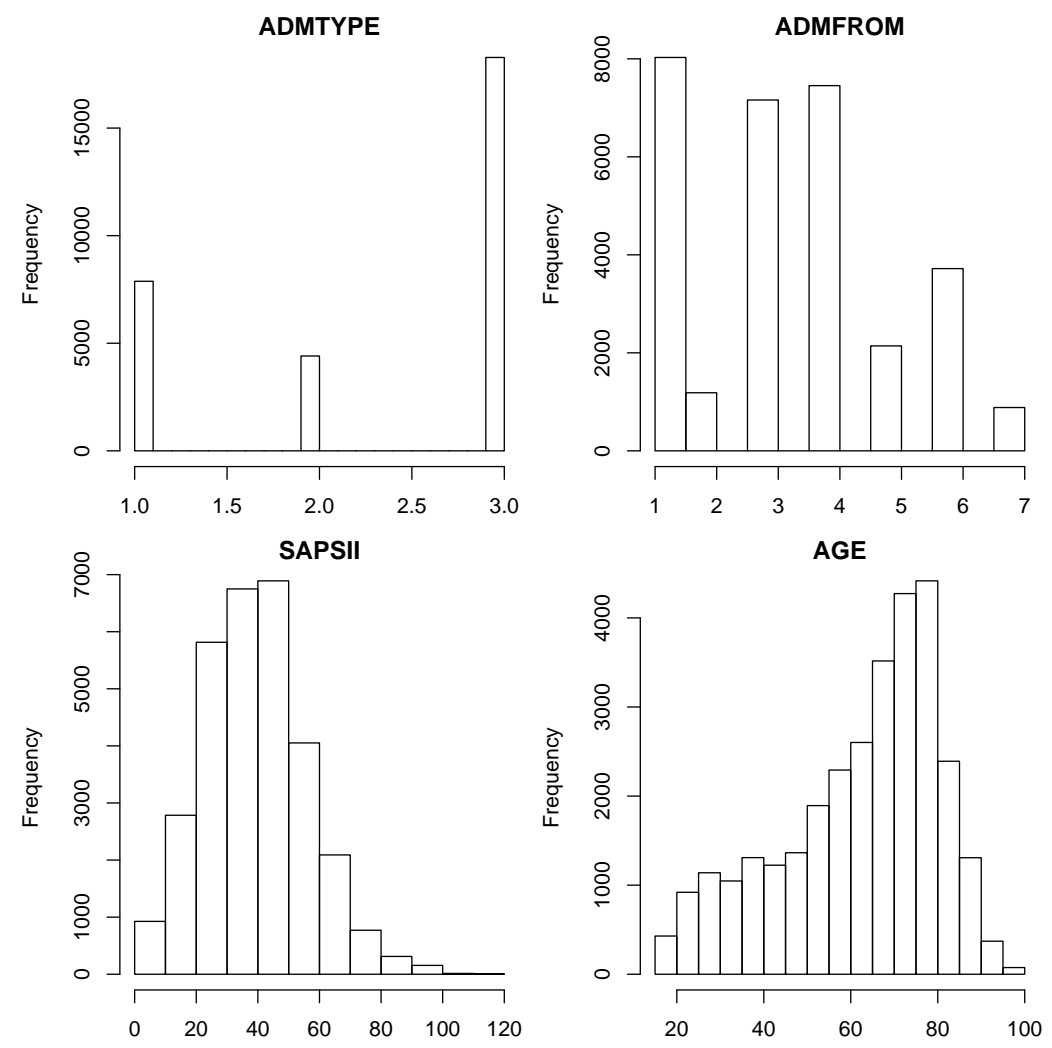

Figure 1: The case mix histograms.

Table 1: The clinical data attributes.

\begin{tabular}{lll}
\hline Attribute & Description & Domain Values \\
\hline respirat & Respiratory & $\{0,1,2,3,4\}$ \\
coagulat & Coagulation & $\{0,1,2,3,4\}$ \\
liver & Liver & $\{0,1,2,3,4\}$ \\
cardiova & Cardiovascular & $\{0,1,2,3,4\}$ \\
cns & Central nervous system & $\{0,1,2,3,4\}$ \\
renal & Renal & $\{0,1,2,3,4\}$ \\
\hline admtype & Admission type & $\{1,2,3\}$ \\
admfrom & Admission origin & $\{1,2, \ldots, 7\}$ \\
SapsII & SAPSII score & $\{0,1, \ldots, 160\}$ \\
age & Patients' age & $\{18,19, \ldots, 100\}$ \\
\hline NBP & Number of daily $B P$ events and critical events & $\{0,1, \ldots, 28\}$ \\
NHR & Number of daily $H R$ events and critical events & $\{0,1, \ldots, 26\}$ \\
NO2 & Number of daily $O 2$ events and critical events & $\{0,1, \ldots, 30\}$ \\
NUR & Number of daily $U R$ events and critical events & $\{0,1, \ldots, 29\}$ \\
\hline
\end{tabular}

the predicted class $\left(P_{k}\right)$ for the $k$ example is given the nearest class value:

$$
P_{k}= \begin{cases}0, & \text { if } s_{k, o}<0.50 \\ 1, & \text { else }\end{cases}
$$

where $s_{k, o}$ denotes the output value for the o output node and the $k$ input example.
Before feeding the Neural Networks, the data was preprocessed: the input values were standardized into the range $[-1,1]$ and a 1 -of- $C$ encoding (one binary variable per class) was applied to the nominal attributes (non ordered) with few categories (SOF $A_{d-1}$, admtype and admfrom). For example, 
Table 2: The event time ranges.

\begin{tabular}{lccc}
\hline Event & $\begin{array}{c}\text { Suggested } \\
\text { Range }\end{array}$ & $\begin{array}{c}\text { Continuously } \\
\text { Out of Range }\end{array}$ & $\begin{array}{c}\text { Intermittently } \\
\text { Out of Range }\end{array}$ \\
\hline$B P(\mathrm{mmHg})$ & $90-180$ & $\geq 10^{\prime}$ & $\geq 10^{\prime}$ in $30^{\prime}$ \\
$O 2(\%)$ & $\geq 90$ & $\geq 10^{\prime}$ & $\geq 10^{\prime}$ in $30^{\prime}$ \\
$H R(\mathrm{bpm})$ & $60-120$ & $\geq 10^{\prime}$ & $\geq 10^{\prime}$ in $30^{\prime}$ \\
$U R(\mathrm{ml} / \mathrm{hour})$ & $\geq 30$ & $\geq 1$ hour & \\
\hline
\end{tabular}

Table 3: The critical event time ranges.

\begin{tabular}{lcccc}
\hline $\begin{array}{l}\text { Critical } \\
\text { Event }\end{array}$ & $\begin{array}{c}\text { Suggested } \\
\text { Range }\end{array}$ & $\begin{array}{c}\text { Continuously } \\
\text { Out of Range }\end{array}$ & $\begin{array}{c}\text { Intermittently } \\
\text { Out of Range }\end{array}$ & $\begin{array}{c}\text { Event } \\
\text { Anytime }\end{array}$ \\
\hline$B P(\mathrm{mmHg})$ & $90-180$ & $\geq 60^{\prime}$ & $\geq 60^{\prime}$ in $120^{\prime}$ & $B P<60$ \\
$O 2(\%)$ & $\geq 90$ & $\geq 60^{\prime}$ & $\geq 60^{\prime}$ in $120^{\prime}$ & $O 2<80$ \\
$H R(\mathrm{bpm})$ & $60-120$ & $\geq 60^{\prime}$ & $\geq 60^{\prime}$ in $120^{\prime}$ & $H R<30 \vee H R>180$ \\
$U R(\mathrm{ml} /$ hour $)$ & $\geq 30$ & $\geq 2$ hours & & $\leq 10$ \\
\hline
\end{tabular}

the admtype variable is fed into 3 input nodes, according to the scheme:

$$
\begin{array}{lrrr}
1 \rightarrow & -1 & -1 & 1 \\
2 \rightarrow & -1 & 1 & -1 \\
3 \rightarrow & -1 & -1 & 1
\end{array}
$$

At the beginning of the training process, the network weights are randomly set within the range $[-1,1]$. Then, the RPROP algorithm (Riedmiller, 1994) is applied, due to its faster convergence and stability, being stopped when the training error slope is approaching zero or after a maximum of $E$ epochs (Prechelt, 1998).

\subsubsection{Statistics}

To insure statistical significance, 30 runs were applied in all tests, being the accuracy estimates achieved using the Holdout method (Flexer, 1996). In each simulation, the available data is divided into two mutually exclusive partitions, using stratified sampling: the training set, used during the modeling phase; and the test set, being used after training, in order to compute the accuracy estimates.

A common tool for classification analysis is the confusion matrix (Kohavi and Provost, 1998), a matrix of size $L \times L$, where $L$ denotes the number of possible classes (domain). This matrix is created by matching the predicted (test result) and actual (patients real condition) values. When $L=2$ and there are four possibilities (Table 4): the number of True Negative (TN), False Positive (FP), False Negative (FN) and True Positive (TP) classifications.

From the matrix, three accuracy measures can be defined (Essex-Sorlie, 1995): the Sensitivity (also known as recall and Type II Error); the Specificity
Table 4: The $2 \times 2$ confusion matrix.

\begin{tabular}{l|cc}
\hline$\downarrow$ actual $\backslash$ predicted $\rightarrow$ & negative & positive \\
\hline negative & $T N$ & $F P$ \\
positive & $F N$ & $T P$ \\
\hline
\end{tabular}

(also known as precision and Type I Error); and the Accuracy, which gives an overall evaluation. These metrics can be computed using the following equations:

$$
\begin{aligned}
& \text { Sensitivity }=\frac{T P}{F N+T P} \times 100(\%) \\
& \text { Specificity }=\frac{T N}{T N+F P} \times 100(\%) \\
& \text { Accuracy }=\frac{T N+T P}{T N+F P+F N+T P} \times 100(\%)
\end{aligned}
$$

\section{Results}

\subsection{Feature Selection}

Four different feature selection configurations will be tested, in order to evaluate the input attribute importance:

A - which uses only the $S O F A_{d-1}$ values (1 variable).

B - where all available input information is used ( $S O F A_{d-1}$ of the corresponding organ system, the case mix and the adverse events, in a total of 9 attributes);

C - in this case, the $S O F A_{d-1}$ is omitted (8 variables); and

D - which uses only the four adverse outcomes. 
Since the SOFA score takes costs and time to obtain, in this study, a special attention will be given to the last two settings.

In the initial experiments, it was considered more important to approach feature selection than model selection. Due to time constrains, the number of hidden nodes was set to round $(N / 2)$, where $N$ denotes the number of input nodes $(N=5, N=21, N=16$ and $N=4$, for the $\mathbf{A}, \mathbf{B}, \mathbf{C}$ and $\mathbf{D}$ setups, respectively); and $\operatorname{round}(x)$ gives nearest integer to the $x$ value.

The commonly used $2 / 3$ and $1 / 3$ partitions were adopted for the training and test sets (Flexer, 1996), while the maximum number of training epochs was set to $E=100$. Each input configuration was tested for all organ systems, being the accuracy measures given in terms of the mean of thirty runs (Table 5).

The A selection manages to achieve a high performance, with an Accuracy ranging from $86 \%$ to $97 \%$, even surpassing the B configuration. This is not surprising, since it is a well established fact that the $S O F A$ is a adequate score for organ dysfunction. Therefore, the results suggest that there is a high correlation between $S O F A_{d-1}$ and $S O F A_{d}$.

When the $S O F A$ index is omitted ( $\mathbf{C}$ and $\mathbf{D})$, the Accuracy values only decay slightly. However, this measure (which is popular within Data Mining community) is not sufficient in Medicine. Ideally, a test should report both high Sensitivity and Specificity values, which suggest a high level of confidence (EssexSorlie, 1995). In fact, there seems to be a tradeoff between these two characteristics, since when the SOF $A$ values are not present (Table 5), the Sensitivity values suffer a huge loss, while the Specificity values increase.

\subsection{Balanced Training}

Why do the A/B selections lead to high Accuracy /Specificity values and low Sensitivity ones? The answer may be due to the biased nature of the organ dysfunction distributions; i.e., there is a much higher number of false (0) than true (1) conditions (Figure $3)$.

One solution to solve this handicap, is to balance the training data; i.e., to use an equal number of true and false learning examples. Therefore, another set of experiments was devised (Table 6), using random sampling training sets, which contained $2 / 3$ of the true examples, plus an equal number of false examples. The test set was composed of the other $1 / 3$ positive entries. In order to achieve a fair comparison with the previous results, the negative test examples were randomly selected from the remaining ones, with a distribution identical to the one found in the original dataset (as given by Figure 3).
The obtained results show a clear improvement in the Sensitivity values, specially for the $\mathbf{C}$ configuration, stressing the importance of the case mix attributes. Yet, the overall results are still far from the ones given by the A selection.

\subsection{Improving Learning}

Until now, the main focus was over selecting the correct training data. Since the obtained results are still not satisfactory, the attention will move towards better Neural Network modeling. This will be achieved by changing two parameters: the number of hidden nodes and the maximum number of training epochs. Due to computational power restrictions, these factors were kept fixed in the previous experiments. However, the adoption of balanced training leads to a considerable reduction of the number of training cases, thus reducing the required training time.

Several experimental trials were conducted, using different combinations of hidden nodes $(H=4,8$, 16 and 32$)$ and maximum number of epochs ( $E=$ 100,500 and 1000), being selected the configuration which gave the lowest training errors $(H=16$ and $E=1000$ ). These setup lead to better results, for all organ systems and accuracy measures (Table 6).

To evaluate the obtained results, a comparison with other Machine Learning classifiers was performed (Table 7), using two classical methods from the WEKA Machine Learning software (Witten and Frank, 2000): Naive Bayes - a statistical algorithm based on probability estimation; and JRIP - a learner based on "IF-THEN" rules.

Although presenting a better Accuracy, the Naive Bayes tends to emphasize the Specificity values, giving poor Sensitivity results. A better behavior is given by the JRIP method, with similar Sensitivity and Specificity values. Nevertheless, the Neural Networks still exhibit the best overall performances.

\section{Conclusions}

The surge of novel bio-inspired tools, such as Neural Networks, has created new exciting possibilities for the field of Clinical Data Mining. In this work, these techniques were applied for organ failure diagnosis of ICU patients.

Preliminary experiments were drawn to test several feature selection configurations, being the best results obtained by the solely use of the SOFA value, measured in the previous day. However, this score takes much more time and costs to be obtained, when compared with the physiologic adverse events. Therefore, another set of experiments were conducted, in order to improve the use of the latter outcomes. First, the 
Table 5: The feature selection performances (in percentage).

\begin{tabular}{lcccccccccccc}
\hline \multirow{2}{*}{ Organ } & \multicolumn{4}{c}{ A } & \multicolumn{4}{c}{ B } & \multicolumn{4}{c}{ C } \\
& Acc. & Sen. & Spe. & Acc. & Sen. & Spe. & Acc. & Sen. & Spe. & Acc. & Sen. & Spe. \\
\hline respirat & 86.3 & 72.4 & 90.2 & 86.2 & 70.0 & 90.8 & 77.9 & 4.4 & 98.8 & 77.6 & 1.8 & 99.4 \\
coagulat & 97.4 & 68.8 & 98.7 & 97.3 & 59.6 & 99.0 & 95.8 & 4.6 & 99.9 & 95.7 & 0.0 & 100 \\
liver & 98.3 & 68.6 & 99.1 & 98.3 & 60.2 & 99.4 & 97.3 & 7.6 & 99.9 & 97.3 & 0.0 & 100 \\
cardiova & 94.2 & 84.1 & 96.3 & 94.2 & 84.0 & 96.3 & 82.8 & 7.5 & 99.0 & 82.2 & 0.5 & 99.8 \\
cns & 95.7 & 92.7 & 96.4 & 95.7 & 92.3 & 96.4 & 83.5 & 23.4 & 97.1 & 81.6 & 0.4 & 99.9 \\
renal & 95.5 & 71.3 & 97.8 & 95.3 & 66.6 & 98.1 & 91.4 & 5.7 & 99.7 & 91.1 & 0.3 & 100 \\
\hline Mean & 94.6 & 76.3 & 96.4 & 94.5 & 72.1 & 96.7 & 88.1 & 8.9 & 99.1 & 87.6 & 0.5 & 99.96 \\
\hline
\end{tabular}

Acc. - Accuracy, Sen. - Sensitivity, Spe - Specificity.

Table 6: The balanced $\mathbf{C}, \mathbf{D}$ and $\mathbf{C}$ improved performances (in percentage).

\begin{tabular}{lccccccccc}
\hline \multirow{2}{*}{ Organ } & \multicolumn{4}{c}{ C } & \multicolumn{4}{c}{ D } & \multicolumn{4}{c}{ C (improved) } \\
& Acc. & Sen. & Spe. & Acc. & Sen. & Spe. & Acc. & Sen. & Spe. \\
\hline respirat & 61.3 & 66.4 & 59.8 & 67.1 & 41.1 & 74.5 & 63.3 & 70.4 & 61.3 \\
coagulat & 67.6 & 66.8 & 67.7 & 73.7 & 41.5 & 75.1 & 70.0 & 72.0 & 69.9 \\
liver & 70.0 & 71.6 & 70.0 & 66.9 & 36.5 & 67.8 & 72.5 & 77.3 & 72.4 \\
cardiova & 65.9 & 62.5 & 66.7 & 68.2 & 37.9 & 74.8 & 69.1 & 66.3 & 69.8 \\
cns & 73.6 & 63.9 & 75.7 & 66.8 & 36.3 & 73.7 & 75.2 & 72.2 & 75.8 \\
renal & 67.8 & 65.6 & 68.0 & 73.2 & 37.6 & 76.6 & 71.9 & 70.5 & 72.0 \\
\hline Mean & 67.7 & 66.2 & 68.0 & 69.3 & 38.5 & 73.8 & 70.3 & 71.5 & 70.2 \\
\hline Acc. - Accuracy, Sen. - Sensitivity, Spe - Specificity.
\end{tabular}

Table 7: The classifiers performances for the $\mathbf{C}$ selection balanced data (in percentage).

\begin{tabular}{lccccccccc}
\hline \multirow{2}{*}{ Organ } & \multicolumn{3}{c}{ Naive Bayes } & \multicolumn{3}{c}{ JRIP } & \multicolumn{3}{c}{ Neural Networks } \\
& Acc. & Sen. & Spe. & Acc. & Sen. & Spe. & Acc. & Sen. & Spe. \\
\hline respirat & 73.5 & 25.2 & 87.3 & 62.8 & 61.9 & 63.0 & 63.3 & 70.4 & 61.3 \\
coagulat & 83.3 & 24.8 & 85.8 & 67.8 & 62.4 & 68.0 & 70.0 & 72.0 & 69.9 \\
liver & 70.8 & 54.3 & 71.2 & 75.7 & 73.7 & 75.7 & 72.5 & 77.3 & 72.4 \\
cardiova & 73.4 & 33.4 & 82.0 & 66.6 & 70.3 & 65.8 & 69.1 & 66.3 & 69.8 \\
cns & 76.3 & 41.3 & 84.2 & 77.6 & 74.4 & 78.3 & 75.2 & 72.2 & 75.8 \\
renal & 76.8 & 45.6 & 79.9 & 69.1 & 68.5 & 69.2 & 71.9 & 70.5 & 72.0 \\
\hline Mean & 75.7 & 37.4 & 81.7 & 69.9 & 68.5 & 70.15 & 70.3 & 71.5 & 70.2 \\
\hline
\end{tabular}

Acc. - Accuracy, Sen. - Sensitivity, Spe - Specificity. 


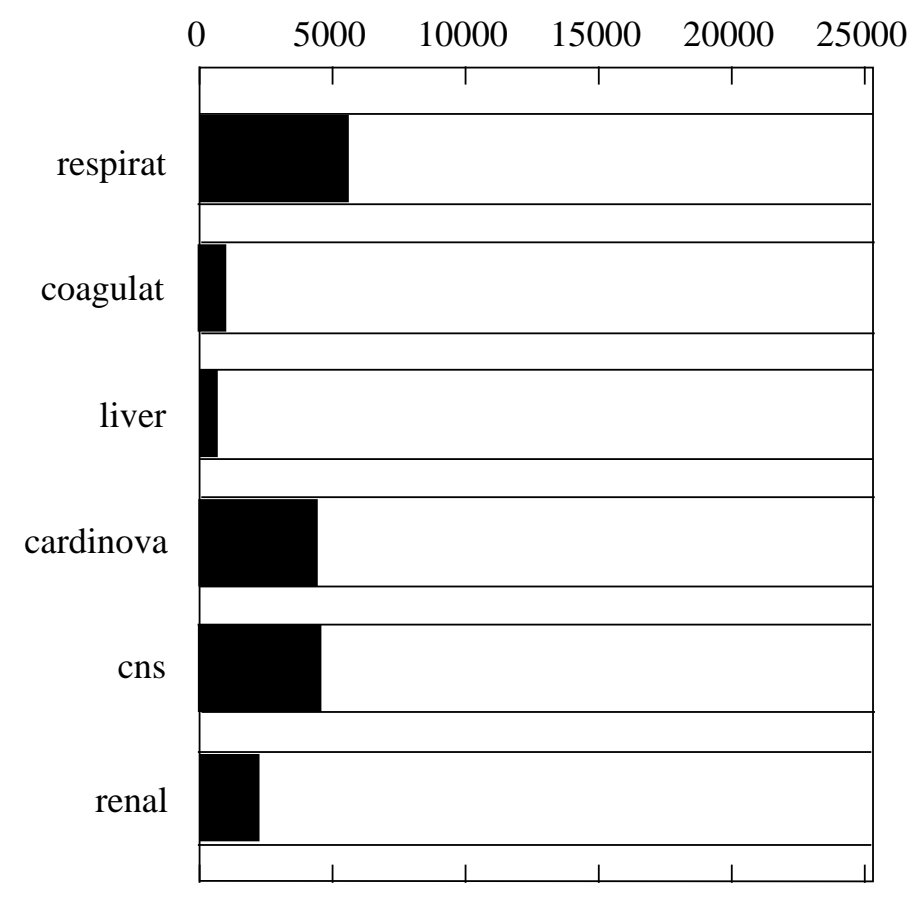

Figure 3: The organ failure true (in black) and false (in white) proportions.

training sets were balanced to contain similar proportions of positive and negative examples. Then, the number of hidden nodes and training epochs was increased. As the result of these changes, an improved performance was gained, specially in terms of sensitivity.

A final comparison between the SOFA score and the proposed solution (the $\mathbf{C}$ improved setup), still favors the former, although the Sensitivity values are close (being even higher for the $\mathbf{C}$ configuration in the coagulation and liver systems). Nevertheless, it is important to stress the main goal of this work: to show that is it possible to diagnose organ failure by using cheap and fast intermediate outcomes (within our knowledge this is done for the first time). The results so far obtained give an overall accuracy of $70 \%$, which although not authoritative, still back this claim. In addiction, the proposed approach opens room for the development of automatic tools for clinical decision support, which are expected to enhance the physician response.

In future research it is intend to improve the performances, by exploring different Neural Network types, such as Radial Basis Functions (Bishop, 1995). Another interesting direction is based in the use of alternative Neural Network training algorithms, which can optimize other learning functions (e.g., Evolutionary Algorithms (Rocha et al., 2003)), since the gradientbased methods (e.g., the RPROP (Riedmiller, 1994)) work by minimizing the Sum Squared Error, a target which does not necessarily correspond to maximizing the Sensitivity and Specificity rates. Finally, it is intended to enlarge the experiments to other ICU applications (e.g., predicting life expectancy).

\section{REFERENCES}

Bishop, C. (1995). Neural Networks for Pattern Recognition. Oxford University Press.

Cios, K. and Moore, G. (2002). Uniqueness of Medical Data Mining. Artifi cial Intelligence in Medicine, 26(12):1-24.

Dybowski, R. (2000). Neural Computation in Medicine: Perspectives and Prospects. In et al., H. M., editor, Proceedings of the ANNIMAB-1 Conference (Artifi cial Neural Networks in Medicine and Biology), pages 26-36. Springer.

Essex-Sorlie, D. (1995). Medical Biostatistics \& Epidemiology: Examination \& Board Review. McGrawHill/Appleton \& Lange, International edition.

Flexer, A. (1996). Statistical evaluation of neural networks experiments: Minimum requirements and current practice. In Proceedings of the 13th European Meeting on Cybernetics and Systems Research, volume 2, pages 1005-1008, Vienna, Austria.

Hand, D., Mannila, H., and Smyth, P. (2001). Principles of Data Mining. MIT Press, Cambridge, MA. 
Haykin, S. (1999). Neural Networks - A Compreensive Foundation. Prentice-Hall, New Jersey, 2nd edition.

Kohavi, R. and Provost, F. (1998). Glossary of Terms. Machine Learning, 30(2/3):271-274.

Prechelt, L. (1998). Early Stopping - but when? In: Neural Networks: Tricks of the trade, Springer Verlag, Heidelberg.

Riedmiller, M. (1994). Supervised Learning in Multilayer Perceptrons - from Backpropagation to Adaptive Learning Techniques. Computer Standards and Interfaces, 16.

Rocha, M., Cortez, P., and Neves, J. (2003). Evolutionary Neural Network Learning. In Pires, F. and Abreu, S., editors, Progress in Artifi cial Intelligence, EPIA 2003 Proceedings, LNAI 2902, pages 24-28, Beja, Portugal. Springer.

Santos, M., Neves, J., Abelha, A., Silva, A., and Rua, F. (2002). Augmented Data Mining Over Clinical Databases Using Learning Classifier System. In Proceedings of the 4th Int. Conf. on Enterprise Information Systems - ICEIS 2002, pages 512-516, Ciudad Real, Spain.

Teres, D. and Pekow, P. (2000). Assessment data elements in a severity scoring system (Editorial). Intensive Care Med, 26:263-264.

Vincent, J., Moreno, R., Takala, J., Willatss, S., Mendonca, A. D., Bruining, H., Reinhart, C., Suter, P., and Thijs, L. (1996). The SOFA (Sepsis-related Organ Failure Assessment) score to describe organ dysfunction / failure. Intensive Care Med, 22:707-710.

Witten, I. and Frank, E. (2000). Data Mining: Practical Machine Learning Tools and Techniques with Java Implementations. Morgan Kaufmann, San Francisco, CA. 